\title{
NMDA Receptor Hypofunction Produces Opposite Effects on Prefrontal Cortex Interneurons and Pyramidal Neurons
}

\author{
Houman Homayoun ${ }^{1,3}$ and Bita Moghaddam ${ }^{1,2}$ \\ Departments of ${ }^{1}$ Neuroscience, ${ }^{2}$ Psychiatry, and ${ }^{3}$ Neurology, University of Pittsburgh, Pittsburgh, Pennsylvania 15260
}

\begin{abstract}
NMDA receptors mediate excitatory postsynaptic potentials throughout the brain but, paradoxically, NMDA receptor antagonists produce cortical excitation in humans and behaving rodents. To elucidate a mechanism for these diverging effects, we examined the effect of use-dependent inhibition of NMDA receptors on the spontaneous activity of putative GABA interneurons and pyramidal neurons in the prefrontal cortex of awake rats. We find that inhibition of NMDA receptors predominately decreases the activity of putative GABA interneurons but, at a delayed rate, increases the firing rate of the majority of pyramidal neurons. Thus, NMDA receptors preferentially drive the activity of cortical inhibitory interneurons suggesting that NMDA receptor inhibition causes cortical excitation by disinhibition of pyramidal neurons. These findings support the hypothesis that NMDA receptor hypofunction, which has been implicated in the pathophysiology of schizophrenia, diminishes the inhibitory control of PFC output neurons. Reducing this effect may be critical for treatment of schizophrenia.
\end{abstract}

Key words: schizophrenia; glutamate; GABA; ensemble unit recording; antipsychotic drugs; metabotropic glutamate receptors

\section{Introduction}

Executive functions of the prefrontal cortex (PFC), including working memory and set-shifting, are critically dependent on NMDA receptors (Krystal et al., 1994; Verma and Moghaddam, 1996; Lisman et al., 1998; Stefani and Moghaddam, 2005). Hypofunction of NMDA receptors is implicated in the cognitive symptoms associated with many brain disorders, especially schizophrenia (Javitt and Zukin, 1991; Olney and Farber, 1995; Coyle, 1996). Oral or intravenous administration of NMDA receptor antagonists such as phencyclidine or ketamine produces transient schizophrenia-like symptoms in healthy individuals (Krystal et al., 1994, 2003; Adler et al., 1999; Newcomer et al., 1999) and impairs PFC-dependent cognitive functions in a manner that is indistinguishable from patients with schizophrenia (Adler et al., 1999). These agents also reinstate the pre-existing symptoms in stabilized patients with schizophrenia (Luby et al., 1959; Lahti et al., 1995b; Malhotra et al., 1997), suggesting that they tap into the same mechanisms that are already compromised in this disorder. These findings have generated great interest in identifying mechanisms by which NMDA receptor hypofunction influences the neuronal activity of the PFC. Animal and clinical work, however, have produced paradoxical results in that, as expected, NMDA antagonists inhibit EPSPs in PFC pyramidal neurons in slices, cultures, and anesthetized preparations (Hirsch and Crepel, 1991; Arvanov and Wang, 1997; Chen et al., 2003), but produce profound cortical activation in functional imaging studies in hu-

\footnotetext{
Received May 15, 2007; revised Sept. 4, 2007; accepted Sept. 4, 2007.

This work was supported by the National Institute of Mental Health.

Correspondence should be addressed to Bita Moghaddam, Department of Neuroscience, University of Pittsburgh, A210 Langley Hall, Pittsburgh, PA 15260. E-mail: bita@pitt.edu.

DOI:10.1523/JNEUROSCI.2213-07.2007

Copyright $\odot 2007$ Society for Neuroscience $\quad$ 0270-6474/07/2711496-05\$15.00/0
}

man volunteers (Lahti et al., 1995a; Breier et al., 1997; Vollenweider et al., 1997; Suzuki et al., 2002) or electrophysiological recording in behaving rodents (Jackson et al., 2004). The latter findings imply that tonic activation of NMDA receptors in an "awake" cortex has principally an inhibitory influence on pyramidal neurons, inconsistent with the conventional view that NMDA-mediated EPSPs contribute to spontaneous activity of these neurons.

In behaving animals, the activity of cortical pyramidal neurons is regulated by fast-spiking (FS) GABA interneurons (Wilson et al., 1994; Markram et al., 2004). These interneurons have faster EPSPs than pyramidal neurons and are more effectively recruited by excitatory inputs (Kawaguchi, 2001; Povysheva et al., 2006), suggesting that NMDA receptors may play a greater role in regulating the spontaneous firing of FS interneurons compared with pyramidal neurons. Thus, a plausible mechanism that might account for NMDA receptor antagonist-induced cortical excitation is that a reduction in NMDA receptor function preferentially reduces the firing rate of FS interneurons, resulting in disinhibition of pyramidal neurons. This will then lead to a net cortical excitation, which is typically observed after NMDA antagonist treatment in human or behaving animal studies (Breier et al., 1997; Vollenweider et al., 1997; Jackson et al., 2004). To test the validity of this mechanism, we compared the effect of a usedependent NMDA antagonist on the spontaneous firing of putative inhibitory interneurons and pyramidal neurons in freely moving rats.

\section{Materials and Methods}

Details of surgical implantation, recording techniques and single-unit isolation have been described previously (Homayoun et al., 2004a; Homayoun and Moghaddam, 2006). Briefly, extracellular single-unit activity was recorded from medial PFC [ (anteroposterior, +2.4-3.6; medio- 
lateral, $+0.5-0.8$; dorsoventral, $-3.5 \mathrm{~mm}$, according to Paxinos and Watson (1998)] of freely moving rats. Extracellular neuronal activity was recorded from chronically implanted electrodes using Recorder software (Plexon, Dallas, TX). Spikes were sorted using Off-Line Sorter (Plexon) and single units were isolated on the basis of discrete waveform clustering, a minimum refractory period of $1.1 \mathrm{~ms}$, stability of their waveform, interspike interval histogram, and autocorrelogram over time (average 2.2 U per electrode). Based on previous work (McCormick et al., 1985; Connors and Gutnick, 1990; Wilson et al., 1994; Tierney et al., 2004), we used the criteria of baseline firing rate $>10 \mathrm{~Hz}$ combined with narrow average spike width to classify FS putative interneurons. FS units also were required to have a high-frequency component in their autocorrelation histograms and interspike interval histograms. Although this double criteria is strongly suggestive of interneurons and unlikely to present pyramidal neurons [regular spiking (RS)], it is possible that a small minority of interneurons could be misclassified as regular firing units. However, such potential misclassification of slow firing interneurons would have minimal impact on the latter group, which is expected to mainly contain the more easily detectable pyramidal neurons.

Data was pooled from 13 rats during 16 recording sessions, in which at least one putative interneuron was recorded per session. (It should be emphasized that putative interneurons were found in relatively few recording sessions and only in a subset of animals. For example, in the same period of time that the present data were collected, no FS units were found in 43 recording sessions.) Animals were injected with the usedependent NMDA receptor antagonist dizocilpine maleate (MK801). The dose of MK801 (0.1 mg/kg, i.p.) was an optimal dose for producing deficits in working memory and set-shifting (Homayoun et al., 2004b; Stefani and Moghaddam, 2005) and increasing spontaneous activity of the majority of RS neurons in the PFC (Jackson et al., 2004). Data were analyzed during a $1 \mathrm{~h}$ window that included a $10 \mathrm{~min}$ baseline period and a 50 min postinjection period. A subset of rats $(n=10 ; 149 \mathrm{RS}$ and $18 \mathrm{FS}$ units) had received a vehicle (saline or distilled water) injection $20 \mathrm{~min}$ before MK801. Another subset ( $n=6 ; 80$ RS and 14 FS units) had no pretreatment. The results obtained in the two subsets were similar in terms of both frequency of responses and their temporal dynamics $(p>$ $0.05)$, and were thus pooled. A total of $6 \mathrm{U}(2.2 \%)$ that did not meet one of the two criteria were excluded. Firing-rate histograms with $1 \mathrm{~min}$ bins (smoothed with a Gaussian filter with bin width of 3) were used to analyze the data. A significant response was defined as 10 consecutive bins crossing the $95 \%$ confidence intervals of the $10 \mathrm{~min}$ baseline, and responses were classified as excitatory, inhibitory or no change. The proportion of response types between groups was compared using $\chi^{2}$ test $(p<0.05)$. Population peridrug firing-rate time histograms were compared across RS and FS groups using two-way ANOVA with time as a repeated measure followed by Bonferroni post hoc test $(p<0.05)$. The onset of a significant response was assessed based on the first $1 \mathrm{~min}$ bin of a 10-bin change compared with baseline. To ensure that the effect of MK801 was not limited to a single dose, recordings were performed in a subset of rats $(n=4)$ with a higher dose of MK801 $(0.3 \mathrm{mg} / \mathrm{kg})$ (for results, see supplemental Fig. 1, available at www.jneurosci.org as supplemental material).

\section{Results}

Single units classified as FS neurons ( $n=32$ from 13 rats) had higher firing rates (Fig. 1 $A$, abscissa, 16.67 vs $3.84 \mathrm{~Hz}$ ) and narrower spike waveforms (Fig. $1 A$, ordinate, peak to valley width, 531.8 vs $272.6 \mu$ s) than RS neurons recorded from the same rats $(n=229)$. Examples of average (Fig. $1 B)$ and composite (Fig. $1 C$ ) waveforms of RS and FS units are depicted. Note that spike waveforms remained stable after MK801 administration (Fig. 1D).

Representative examples of individual responses of FS and RS neurons (Fig. $2 A, B$ ) demonstrate divergent responses to systemic administration of MK801. FS neurons primarily displayed an inhibitory response whereas the majority of RS neurons had a profound excitatory response to MK801. As shown in Figure 2C, $\sim 69 \%$ of FS neurons decreased their firing rate whereas $86 \%$ of RS, recorded during the same sessions, increased their firing rate

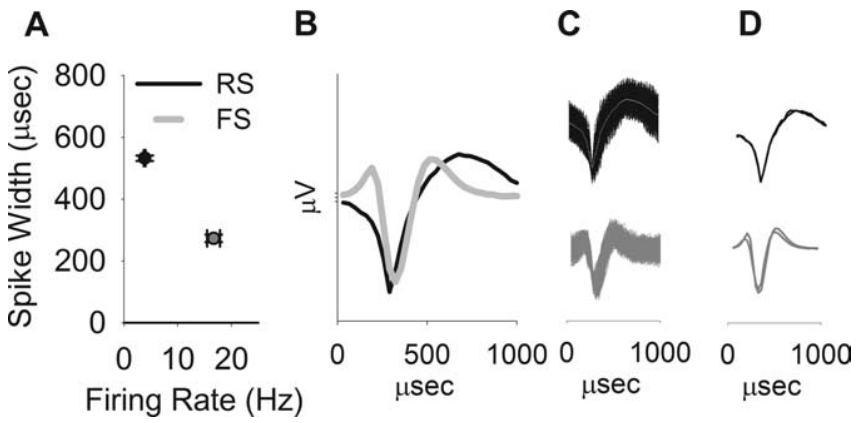

Figure 1. Distinction between putative interneurons (FS units) and pyramidal neurons (RS units). $\boldsymbol{A}$, The population of putative interneurons had a higher firing rate (abscissa) and narrower spikeform (ordinate, average \pm SEM of peak to valley spike width). $\boldsymbol{B}$, Average waveforms of representative RS and FS units. $C$, Superimposed composite of all the waveforms for the same units. D, Superimposed average waveforms during two 10 min windows, pre- and postMK801 administration. The waveforms remained stable after MK801 treatment compared with the predrug period.

after MK801. A $\chi^{2}$ test indicated a significant difference in distribution of responses to MK801 between populations of pyramidal neurons and interneurons (Fig. $2 C)\left(\chi^{2}=100.42 ; p<0.001\right)$. The population activity in response to MK801 showed a sustained average inhibitory response by FS neurons and excitatory response by RS neurons (Fig. 2D). Two-way ANOVA with time as repeated measure revealed a significant difference between average firing rate time histograms of two types of units (type, $F_{(1,259)}=12.96, p<0.001$; time, $F_{(59,15281)}=2.71, p<0.001$; type by time interaction, $\left.F_{(59,15281)}=5.94, p<0.001\right)$. Furthermore, the predominant inhibitory effect on FS units was not an artifact of the dose of MK801 and was repeated using a higher dose of MK801 $(0.3 \mathrm{mg} / \mathrm{kg})$ in a distinct set of animals (supplemental Fig. 1, available at www.jneurosci.org as supplemental material).

Visual comparison of individual neuronal responses (Fig. $3 A, B$ ) and the population histograms (Fig. 2D) suggested that FS neurons started to inhibit their firing and reached their inhibitory plateau in advance of the onset and plateau of the excitatory response of the simultaneously recorded RS units. This observation was verified by plotting the delay to onset/plateau of the inhibitory response of interneurons recorded within each session against the average excitatory onset/plateau of concurrently recorded pyramidal cells (Fig. $3 C$, onset, linear regression analysis, $r^{2}=0.95, p<0.001, D$, plateau, $r^{2}=0.94, p<0.001$, insets, interval between corresponding inhibitory and excitatory onset and plateau).

\section{Discussion}

These findings indicate that in an awake cortex, NMDA receptors preferentially regulate the firing rate of cortical GABA interneurons. The impact of NMDA receptor inhibition on pyramidal neuron activity was profoundly excitatory, suggesting that this effect is indirect and caused by disinhibition of GABA neurons that control the firing of pyramidal neurons. This conclusion is consistent with the observation that inhibition of putative interneurons by the NMDA receptor antagonist started earlier and reached its plateau before the excitation of pyramidal neurons. It should be emphasized, however, that the relatively long delay between the start/plateau of inhibitions and excitations in our sampled neuronal population suggests that blocking the NMDA receptors is influencing complex network dynamics that affect the firing rate of FS and RS neurons for sustained periods of time. This is consistent with a role for NMDA receptors in mediating 

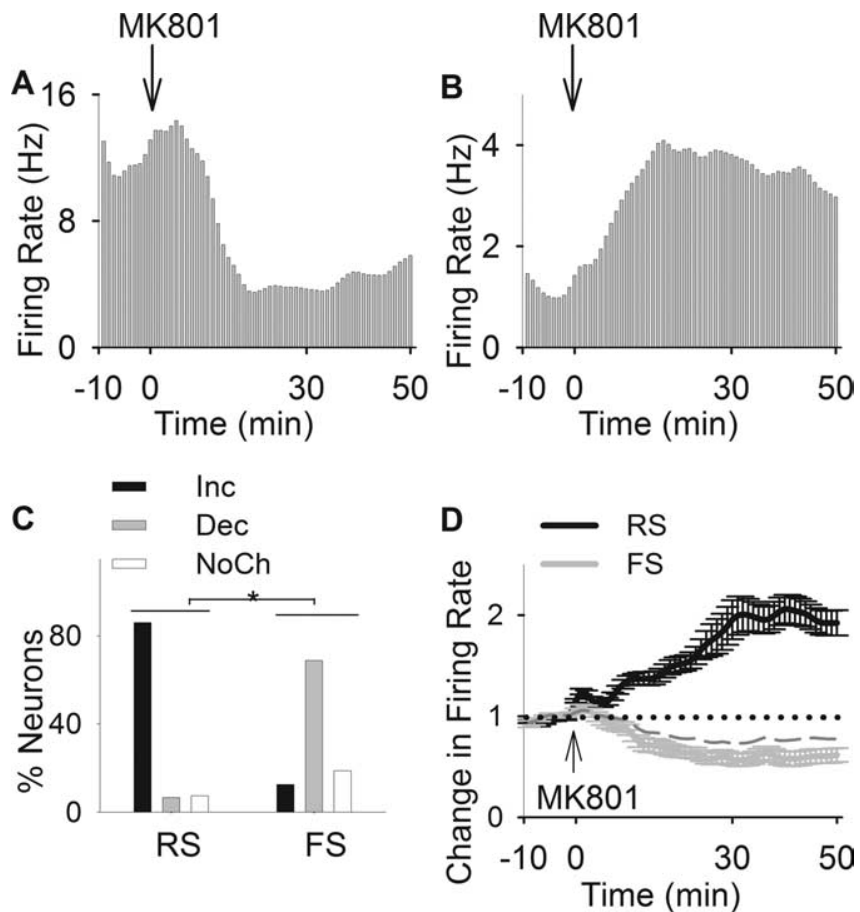

Figure 2. Inhibitory effects of MK801 on putative interneurons. $A, B$, Examples of inhibitory and excitatory effects of MK801 on an individual interneuron $(\boldsymbol{A})$ and a pyramidal neuron $(\boldsymbol{B})$. Each panel depicts the firing rate histogram (bin, 1 min; smoothed with a Gaussian filter with bin width of 3) of a single unit. Arrow indicates the time of MK801 injection. C, Distribution of firing rate responses (increase, decrease, or no change) to MK801 was significantly different among populations of RS and FS units $\left({ }^{*} p<0.001\right)$. $D$, Comparison of average population response of RS and FS units to MK801. Two-way ANOVA with time as the repeated measure revealed a significant effect for neuron type $\left(F_{(1,259)}=12.96 ; p<0.001\right)$, time $\left(F_{(59,15281)}=\right.$ $2.71 ; p<0.001)$, and type by time interaction $\left(F_{(59,15281)}=5.94 ; p<0.001\right)$. The averages for all FS units and those with a decreased response type have been depicted as dashed and solid gray lines, respectively.

excitatory reverberation and various network models that incorporate NMDA receptors in maintaining integrator circuits or temporal accumulation of spike activity that correlate with behavioral events (Koulakov et al., 2002; Wong and Wange, 2006).

Synaptic inhibition is necessary for spatial tuning of pyramidal neurons and synchronized firing of cortical networks and, thus, is thought to play a critical role in the "executive" functions associated with these regions (Goldman-Rakic, 1999; Kawaguchi, 2001; Markram et al., 2004). For example, blocking GABA receptors in the PFC and the resulting disinhibition of pyramidal neurons disrupts the tuning of these neurons during sensorimotor and mnemonic phases of a working memory task (Rao et al., 2000; Constantinidis and Goldman-Rakic, 2002). The present findings suggest that the activity of cortical GABA interneurons is highly sensitive to tonic regulation by NMDA receptors. The basal activity of pyramidal neurons, in contrast, was not directly regulated by NMDA receptors, but was susceptible to disinhibition. This concomitant interneuron inhibition-pyramidal disinhibition process supports the hypothesis that reduced NMDA function produces cortical excitation and impair PFC-dependent cognitive functions by disrupting inhibitory control of pyramidal neurons.

The PFC contains several different types of GABAergic interneurons, which are classified based on morphology and expression of calcium-binding proteins (Markram et al., 2004). Interneurons that express calbindin- or calretinin-binding proteins primarily target the distal dendrites of pyramidal neurons whereas interneurons that express parvalbumin (PV) synapse
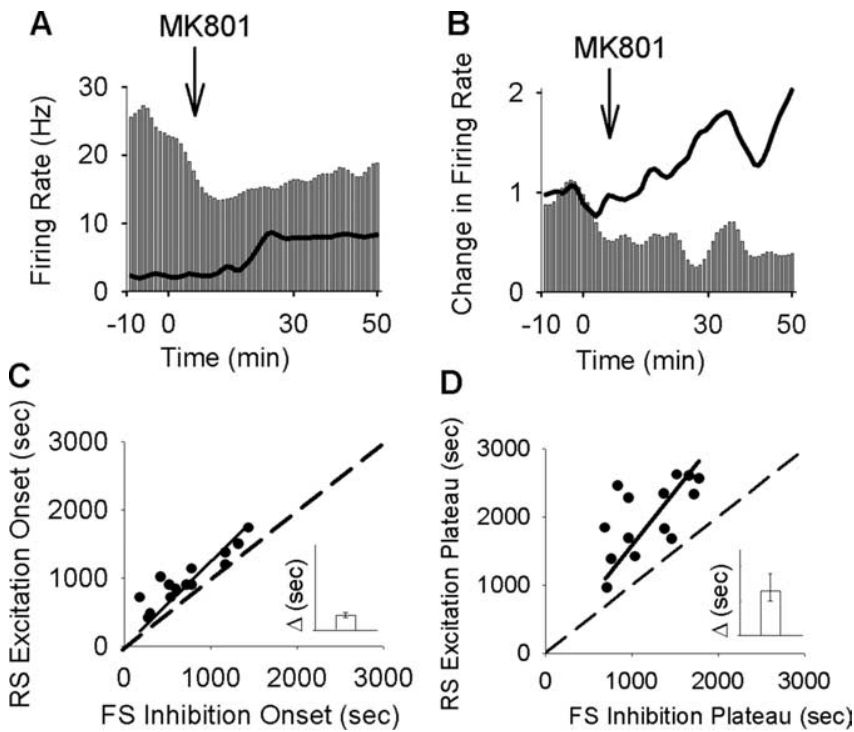

Figure 3. MK801 inhibition of interneurons precedes its excitation of pyramidal cells. $\boldsymbol{A}$, Superimposed firing-rate histogram of simultaneously recorded pairs of interneurons (bar graph) and pyramidal neurons (solid line). Arrow indicates the time of MK801 injection. Bin size, $1 \mathrm{~min}$. $\boldsymbol{B}$, Normalized firing rate histogram of another pair of simultaneously recorded interneuron (bar graph; baseline firing rate, $17.26 \mathrm{~Hz}$ ) and pyramidal neuron (solid line; baseline, 2.86 $\mathrm{Hz}$ ). Inset, Finer timescale with $20 \mathrm{~s}$ bins. C, D, Putative interneurons preceded their concurrently recorded pyramidal neurons both in the onset of their inhibition $(\boldsymbol{C})$ and in reaching the inhibitory plateau $(\boldsymbol{D})$. Each dot in the scatter plots depicts the post-MK801 delay to onset $(\boldsymbol{C})$ or plateau $(\boldsymbol{D})$ of inhibitory response for interneuron/s recorded during a single session versus the delay to onset $(\boldsymbol{C})$ or plateau $(\boldsymbol{D})$ of excitatory response for the average pyramidal neurons in the same session. A dashed line indicates no change from baseline and a solid line depicts the linear regression. Inset, Median and 95\% confidence intervals of the difference in the delay between inhibitory versus excitatory response onset $(\boldsymbol{C})$ or plateau $(\boldsymbol{D})$, with a positive value indicating inhibition preceding the excitation ( $y$-axis range, $0-1500$ s).

primarily on the axon initial segment and cell body proximal dendrites. The PV-containing interneurons are, therefore, in a better position to regulate the firing rate of pyramidal neurons. These neurons receive extensive excitatory input, which accounts for their high basal firing rates, and are sensitive to neurotoxic effects of NMDA receptor antagonists during development (Kawaguchi and Kubota, 1993; Jones and Buhl, 1999; Cochran et al., 2003; Goldberg et al., 2003). They have wide-spread axonal arborization with a single cell estimated to synapse onto $>200$ pyramidal neurons, thus inhibiting the firing rate of large groups of cortical afferents (Cobb et al., 1995). Because of methodological limitations associated with awake animal recording, we could not identify the class of FS neurons in our study; however, based on the fast baseline firing rate of theses neurons (Kawaguchi and Kubota, 1993), it may be hypothesized that the majority of them were PV-containing interneurons. This would explain the profound influence that inhibition of putative FS neurons had on disinhibiting the majority of pyramidal neurons recorded from the same electrode array.

Functional deficits in GABA interneurons have been reported in schizophrenia (Akbarian et al., 1995; Lewis et al., 2005), suggesting that NMDA receptor antagonists mimic the symptoms of schizophrenia by producing transient functional deficits in GABA interneurons. The NMDA hypofunction paradigm for modeling schizophrenia possesses both face validity, as it closely replicates a wide spectrum of schizophrenia-like symptoms (Krystal et al., 1994; Adler et al., 1999), and predictive validity, as it distinguishes between atypical and typical antipsychotics based on their ability to suppress abnormal PFC activity (Homayoun 
and Moghaddam, 2007), and has been the basis for discovery of a novel antipsychotic drug (Moghaddam and Adams, 1998; Patil et al., 2007). The construct validity of this model, however, has been limited to a wide variety of schizophrenia susceptibility genes encoding for proteins that could regulate the function of NMDA receptors (Moghaddam, 2003; Harrison and Weinberger, 2005). The present observation adds an important aspect to the construct validity of this paradigm by demonstrating that it is associated with reduced activity of putative GABA interneurons in the PFC. Thus, the hypothesized NMDA receptor hypofunction results in functional deficits of cortical GABA interneurons, which is a consistent pathological finding in schizophrenia (Coyle, 2004; Lewis et al., 2005). More importantly, our findings provide a clinically relevant mechanism where the resulting hyperactivity of pyramidal neurons (Jackson et al., 2004) and excessive glutamate spillover (Moghaddam et al., 1997) can be the basis for discovery of novel antipsychotic drugs (Moghaddam and Adams, 1998; Patil et al., 2007).

\section{References}

Adler CM, Malhotra AK, Elman I, Goldberg T, Egan M, Pickar D, Breier A (1999) Comparison of ketamine-induced thought disorder in healthy volunteers and thought disorder in schizophrenia. Am J Psychiatry 156:1646-1649.

Akbarian S, Kim JJ, Potkin SG, Hagman JO, Tafazzoli A, Bunney Jr WE, Jones EG (1995) Gene expression for glutamic acid decarboxylase is reduced without loss of neurons in prefrontal cortex of schizophrenics. Arch Gen Psychiatry 52:258-266.

Arvanov VL, Wang RY (1997) NMDA-induced response in pyramidal neurons of the rat medial prefrontal cortex slices consists of NMDA and non-NMDA components. Brain Res 768:361-364.

Breier A, Malhotra AK, Pinals DA, Weisenfeld NI, Pickar D (1997) Association of ketamine-induced psychosis with focal activation of the prefrontal cortex in healthy volunteers. Am J Psychiat 154:805-811.

Chen L, Muhlhauser M, Yang CR (2003) Glycine tranporter-1 blockade potentiates NMDA-mediated responses in rat prefrontal cortical neurons in vitro and in vivo. J Neurophysiol 89:691-703.

Cobb SR, Buhl EH, Halasy K, Paulsen O, Somogyi P (1995) Synchronization of neuronal activity in hippocampus by individual GABAergic interneurons. Nature 378:75-78.

Cochran SM, Fujimura M, Morris BJ, Pratt JA (2003) Acute and delayed effects of phencyclidine upon mRNA levels of markers of glutamatergic and GABAergic neurotransmitter function in the rat brain. Synapse 46:206-214.

Connors BW, Gutnick MJ (1990) Intrinsic firing patterns of diverse neocortical neurons. Trends Neurosci 13:99-104.

Constantinidis C, Goldman-Rakic P (2002) Correlated discharges among putative pyramidal neurons and interneurons in the primate prefrontal cortex. J Neurophysiol 88:3487-3497.

Coyle JT (1996) The glutamatergic dysfunction hypothesis for schizophrenia. Harvard Rev Psychiatry 3:241-253.

Coyle JT (2004) The GABA-glutamate connection in schizophrenia: which is the proximate cause? Biochem Pharmacol 68:1507-1514.

Goldberg JH, Yuste R, Tamas G (2003) $\mathrm{Ca}^{2+}$ imaging of mouse neocortical interneurone dendrites: contribution of $\mathrm{Ca}^{2+}$-permeable AMPA and NMDA receptors to subthreshold $\mathrm{Ca}^{2+}$ dynamics. J Physiol (Lond) 551:67-78.

Goldman-Rakic P (1999) The "psychic" neuron of the cerebral cortex. Ann NY Acad Sci 868:13-26.

Harrison P, Weinberger D (2005) Schizophrenia genes, gene expression, and neuropathology: on the matter of their convergence. Molecular Psychiat 10:40-68.

Hirsch JC, Crepel F (1991) Blockade of NMDA receptors unmasks a longterm depression in synaptic efficacy in rat prefrontal neurons in vitro. Exp Brain Res 85:621-624.

Homayoun H, Moghaddam B (2006) Progression of cellular adaptations in medial prefrontal and orbitofrontal cortex in response to repeated amphetamine. J Neurosci 26:8025-8039.

Homayoun H, Moghaddam B (2007) Fine-tuning of awake prefrontal cor- tex neurons by clozapine: comparison with haloperidol and $N$-desmethylclozapine. Biol Psychiatry 61:679-687.

Homayoun H, Jackson ME, Moghaddam B (2004a) Activation of metabotropic glutamate 2/3 (mGlu2/3) receptors reverses the effects of NMDA receptor hypofunction on prefrontal cortex unit activity in awake rats. J Neurophysiol 93:1989-2001.

Homayoun H, Stefani MR, Adams BW, Tamagan GD, Moghaddam B (2004b) Functional interaction between NMDA and mGlu5 receptors: effects on working memory, instrumental learning, motor behaviors, and dopamine release. Neuropsychopharmacology 29:1259-1269.

Jackson M, Homayoun H, Moghaddam B (2004) NMDA receptor hypofunction produces concomitant firing rate potentiation and burst activity reduction in the prefrontal cortex. Proc Natl Acad Sci USA 101:6391-6396.

Javitt DC, Zukin SR (1991) Recent advances in the phencyclidine model of schizophrenia. Am J Psychiatry 148:1301-1308.

Jones RS, Buhl EH (1999) Basket-like interneurones in layer II of the entorhinal cortex exhibit a powerful NMDA-mediated synaptic excitation. Neurosci Lett 149:35-39.

Kawaguchi Y (2001) Distinct firing patterns of neuronal subtypes in cortical synchronized activities. J Neurosci 21:7261-7272.

Kawaguchi Y, Kubota Y (1993) Correlation of physiological subgroupings of nonpyramidal cells with parvalbumin- and calbindinD28kimmunoreactive neurons in layer $\mathrm{V}$ of rat frontal cortex. J Neurophysiol 70:387-396.

Koulakov AA, Raghavachari S, Kepecs A, Lisman JE (2002) Model for a robust neural integrator. Nat Neurosci 5:775-782.

Krystal JH, Karper LP, Seibyl JP, Freeman GK, Delaney R, Bremner JD, Heninger GR, Bowers Jr M, Charney DS (1994) Subanesthetic effects of the noncompetitive NMDA antagonist, ketamine, in humans: psychotomimetic, perceptual, cognitive, and neuroendocrine responses. Arch Gen Psychiatry 51:199-214.

Krystal JH, D’Souza DC, Mathalon D, Perry E, Belger A, Hoffman R (2003) NMDA receptor antagonist effects, cortical glutamatergic function, and schizophrenia: toward a paradigm shift in medication development. Psychopharmacology (Berl) 169:215-233.

Lahti AC, Holocomb HH, Medoff DR, Tammings CA (1995a) Ketamine activates psychosis and alters limbic blood flow in schizophrenia. NeuroReport 6:869-872.

Lahti AC, Koffel B, LaPorte D, Tamminga CA (1995b) Subanesthetic doses of ketamine stimulate psychosis in schizophrenia. Neuropsychopharmacology 13:9-19.

Lewis DA, Hashimoto T, Volk DW (2005) Cortical inhibitory neurons and schizophrenia. Nat Rev Neurosci 6:312-324.

Lisman J, Fellous J, Wang X (1998) A role for NMDA-receptor channels in working memory. Nat Neurosci 1:273-275.

Luby E, Cohen B, Rosenbaum G, Gottlieb J, Kelley R (1959) Study of a new schizophrenomimetic drug; sernyl. Am Med Assoc Arch Neurol Psychiatry 81:363-369.

Malhotra AK, Pinals DA, Adler CM, Elman I, Clifton A, Pickar D, Breier A (1997) Ketamine-induced exacerbation of psychotic symptoms and cognitive impairment in neuroleptic-free schizophrenics. Neuropsychopharmacology 17:141-150.

Markram H, Toledo-Rodriguez M, Wang Y, Gupta A, Silberberg G, Wu C (2004) Interneurons of the neocortical inhibitory system. Nat Rev Neurosci 5:793-807.

McCormick DA, Connors BW, Lighthall JW, Prince DA (1985) Comparative electrophysiology of pyramidal and sparsely spiny stellate neurons of the neocortex. J Neurophysiol 54:782-806.

Moghaddam B (2003) Bringing order to the glutamate chaos in schizophrenia. Neuron 40:881-884.

Moghaddam B, Adams B (1998) Reversal of phencyclidine effects by a group II metabotropic glutamate receptor agonist in rats. Science 281:1349-1352.

Moghaddam B, Adams B, Verma A, Daly D (1997) Activation of glutamatergic neurotransmission by ketamine: a novel step in the pathway from NMDA receptor blockade to dopaminergic and cognitive disruptions associated with the prefrontal cortex. J Neurosci 17:2921-2927.

Newcomer JW, Farber NB, Jevtovic-Todorovic V, Selke G, Melson AK, Hershey T, Craft S, Olney JW (1999) Ketamine-induced NMDA receptor hypofunction as a model of memory impairment and psychosis. Neuropsychopharmacology 20:106-118. 
Olney J, Farber N (1995) Glutamate receptor dysfunction and schizophrenia. Arch Gen Psychiatry 52:998-1007.

Patil ST, Zhang L, Martenyi F, Lowe SL, Jackson KA, Andreev BV, Avedisova AS, Bardenstein LM, Gurovich IY, Morozova MA, Mosolov SN, Neznanov NG, Reznik AM, Smulevich AB, Tochilov VA, Johnson BG, Monn JA, Schoepp DD (2007) Activation of mGlu2/3 receptors as a new approach to treat schizophrenia: a randomized Phase 2 clinical trial. Nat Med 13:1102-1107.

Paxinos G, Watson C (1998) The rat brain in stereotaxic coordinates. San Diego: Academic.

Povysheva NV, Gonzalez-Burgos G, Zaitsev AV, Kroner S, Barrionuevo G, Lewis DA, Krimer LS (2006) Properties of excitatory synaptic responses in fast-spiking interneurons and pyramidal cells from monkey and rat prefrontal cortex. Cereb Cortex 16:541-552.

Rao A, Cha EM, Craig AM (2000) Mismatched appositions of presynaptic and postsynaptic components in isolated hippocampal neurons. J Neurosci 20:8344-8353.

Stefani MR, Moghaddam B (2005) Systemic and prefrontal cortical NMDA receptor blockade differentially affect discrimination learning and setshift ability in rats. Behav Neurosci 119:420-428.
Suzuki Y, Jodo E, Takeuchi S, Niwa S, Kayama Y (2002) Acute administration of phencyclidine induces tonic activation of medial prefrontal cortex neurons in freely moving rats. Neurosci 114:769-779.

Tierney P, Degenetais E, Thierry AM, Glowinski J, Gioanni Y (2004) Influence of the hippocampus on interneurons of the rat prefrontal cortex. Eur J Neurosci 20:514-524.

Verma A, Moghaddam B (1996) NMDA receptor antagonists impair prefrontal cortex function as assessed via spatial delayed alternation performance in rats: modulation by dopamine. J Neurosci 16:373-379.

Vollenweider FX, Leenders KL, Oye I, Hell D, Angst J (1997) Differential psychopathology and patterns of cerebral glucose utilisation produced by (S)- and (R)-ketamine in healthy volunteers using positron emission tomography (PET). Eur Neuropsychopharmacol 7:25-38.

Wilson FA, O'Scalaidhe SP, Goldman-Rakic PS (1994) Functional synergism between putative $\gamma$-aminobutyrate-containing neurons and pyramidal neurons in prefrontal cortex. Proc Natl Acad Sci USA 91:4009-4013.

Wong KF, Wange XJ (2006) A recurrent network mechanism of time integration in perceptual decisions. J Neurosci 26:1314-1328. 